\title{
L'ÉVALUATION CIRCULAIRE : UN MODÈLE SYSTÉMIQUE POUR PERMETTRE AUX ORGANISATIONS HUMAINES DE S'INSCRIRE DANS LA DURABILITÉ
}

\author{
Author(s) / Auteur(s) : \\ Piecq Andrée \\ Secrétaire générale de l'Union européenne de systémique (UES) \\ Directrice scientifique de l'Institut Indépendant de Systémique des organisations G.I.R.O.S. \\ a.piecq@gmail.com
}

\begin{abstract}
Résumé :
Le modèle systémique - «L'évaluation circulaire »-a été élaboré sur le plan théorique et a été mis en pratique. Son objectif est d'inscrire toutes les organisations marchandes, non marchandes ou administratives dans la durabilité. A intervalles réguliers, il accède à leur structure pour diagnostiquer leur fonctionnement. Il a été élaboré par Andrée Piecq dans le cadre de ses travaux centrés sur les organisations humaines.

L'" évaluation circulaire" est un processus qui, grâce à des observations multipliées dans le temps du fonctionnement de l'organisation, empêche l'apparition de spirales négatives. En effet, ce sont ces spirales qui menacent la durabilité de l'organisation.

Ce processus s'inscrit dans :

- une évaluation permanente ;

- la prise en compte des contextes, y compris celui du temps ;

- l'analyse des douze " principes directeurs »;

- la mise en exergue les symptômes de bon fonctionnement ou de dysfonctionnement ;

- l'élaboration du diagnostic du fonctionnement de l'organisation ;

- l'élaboration des stratégies de changement/non changement qui répondent à la finalité de l'organisation.
\end{abstract}

En conclusion ce processus permet l'augmentation de la créativité de l'organisation et la conduit à s'inscrire dans la durabilité.

Keywords / Mots-clés :

évaluation circulaire, principes directeurs, diagnostics, stratégies de changement, durabilité

\section{INTRODUCTION}

L'observation de notre civilisation montre qu'elle a atteint un seuil de complexité inégalée et que pouvoir élaborer des stratégies face aux aléas qu'elle rencontre est devenu très difficile.

Les organisations sont attaquées de plein fouet par les pressions de l'environnement techniques, sociales et financières. Ces pressions les confrontent à des changements permanents de contextes et les obligent à évoluer. Une de leur principale priorité est devenue le maintien de leur cap pour s'inscrire dans la durabilité et développer leur capacité de résilience organisationnelle.

Un modèle systémique, «l'évaluation circulaire », est une réponse, tant théorique que pratique, à cette priorité. C'est un processus permanent qui évalue le fonctionnement des organisations afin d'élaborer des stratégies qui empêcheront l'apparition de spirales négatives et permettront à l'organisation de s'inscrire dans la durabilité.

\section{COMMENT ÉVALUER LE FONCTIONNEMENT DES ORGANISATIONS ?}

Evaluer le fonctionnement des Organisations se fait à l'aide du «Giroscope » (Piecq, 2011), un modèle systémique basé sur l'observation de l'ensemble des "comportements » de l'organisation (écrits, entretiens, des faits...). Ces comportements s'inscrivent dans les contextes dans lesquels l'organisation s'inscrit. 
A partir de là, des hypothèses sont élaborées sur les «principes directeurs » mis en jeu. A savoir système/sous-système, membres, finalité, frontières, règles, circularité, totalité, émission d'informations, réceptions d'informations, rétroaction, homéostasie, équifinalité (Piecq, 2011).

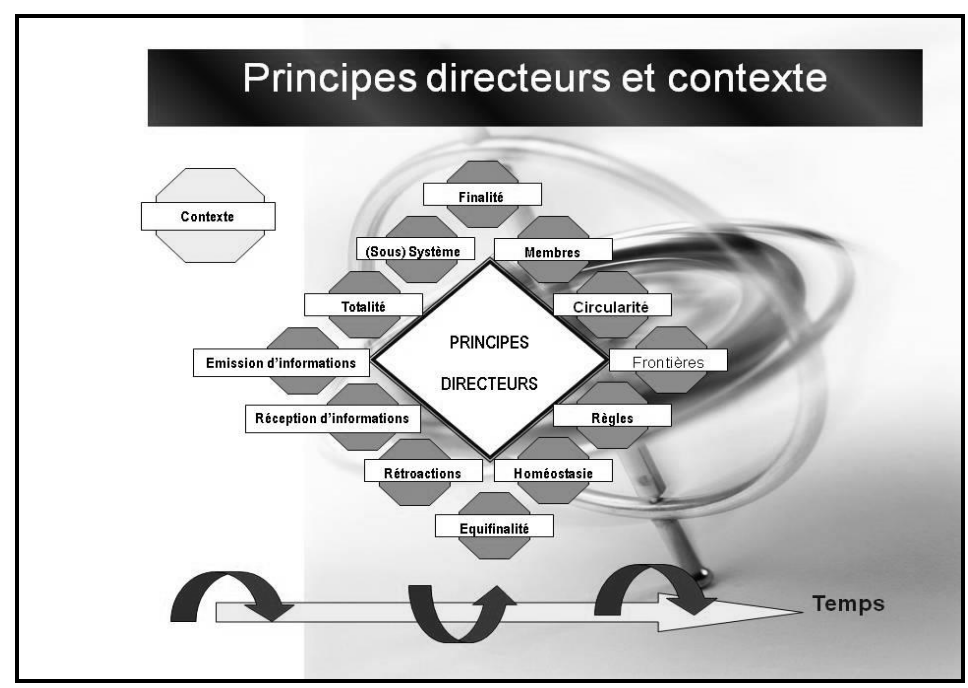

Ils sont en interactions, les uns avec les autres et avec les contextes de l'organisation et forment la structure de l'organisation. A partir de là, un diagnostic du fonctionnement de l'organisation est élaboré en termes de fonctionnement opérant ou inopérant. Des stratégies de types différents sont alors élaborées.

\section{LES TYPES DE STRATÉGIES MISES EN PLACE}

Elaborer des stratégies, c'est trouver le moyen d'introduire le changement mais en quoi consiste le changement?

Pour Watzlawick (Fisch, Weakland, Watzlawick, 1975), il existe deux types de changement, le changement 1 et le changement 2 qui qualifient l'état dans lequel le système se trouve après la mise en place de tentatives de résolution de ses difficultés.

Le changement 1 ne modifie pas la structure de l'organisation :

- Les interactions se positionnent à l'intérieur des principes directeurs mais pas entre eux

- Ce sont des changements purement correctifs

Le changement 2 modifie la structure de l'organisation :

Les interactions entre les principes directeurs, et avec, les contextes sont modifiés et conduisent à une déviation de la norme et la nouvelle structure émerge. Pour rappel, Ilya Prigogine (Prigogine \& Stengers, 1986) a écrit : "Sans la déviation de la norme, la discontinuité n'existe pas, et une nouvelle structure ne peut émerger".

Lorsqu'une organisation (une entreprise) rencontre des difficultés de quel qu'ordre qu'elles soient, les étapes préalables à suivre pour élaborer des stratégies de changement sont :

- L'observation et la formulation d'hypothèses sur les PDI, et l'établissement du diagnostic en fonctionnement opérant ou inopérant.

- La vérification des hypothèses par un questionnement.

- L'élaboration du diagnostic du fonctionnement en opérant ou inopérant

- L'approfondissement des sources présumées des difficultés présentées par l'organisation. 
- Le Dépistage des pseudos changements qui suppriment seulement le(s) symptôme(s) observé(s) sans modifier la structure même du système (les interactions).

Ces étapes permettent de pouvoir définir quelle stratégie mettre en place.

Si le fonctionnement de l'organisation est qualifié d'opérant, le conduire vers un changement de type 1 sera suffisant. Ces stratégies sont des AMENAGEMENTS.

Si le fonctionnement est qualifié d'inopérant le changement doit être de type 2. Ces stratégies sont des MUTATIONS.

\subsection{L'aménagement}

Ce sont des stratégies mises facilement en place après leur dépistage. En effet, ce sont des changements purement correctifs qui ne modifient pas la structure de l'organisation

Elles nécessitent $\mathrm{du}$ «bon sens » et sont logiques.

\section{Face à une difficulté $\mathrm{A}$ la stratégie sera non $\mathrm{A}$}

Mais changer une difficulté en son contraire si on n'en vérifie pas l'impact sur l'ensemble des PDI, peut amener une difficulté plus grande allant même jusqu'à faire émerger un changement de structure qui conduira à un fonctionnement qui peut devenir inopérant.

\subsection{La Mutation}

Ce sont des stratégies élaborées pour sortir de situations inextricables. Des aménagements ne permettent pas d'en sortir ou peuvent même faire empirer la situation. L'organisation doit être amenée à changer de structure en :

- Vérifiant les interactions entre les membres de l'organisation (départements, membres individuels), l'organisation et la situation.

- Tenant compte de ce qui ne doit absolument pas être modifié, car cela a déjà été expérimenté.

- Accédant au principe directeur qui sera le levier du changement.

Pour qu'il y ait mutation face à une difficulté $\mathrm{A}$, il faut avoir à la fois non $\mathrm{A}$ mais aussi pas non $\mathrm{A}$.

Le choix est rejeté, la modification se porte sur un autre PDI ce qui conduit à une transformation de la structure.

Les deux types stratégies peuvent avoir comme analogie des modifications faites dans des bâtiments :

- Soit il s'agit d'une modification interne qui consiste à peindre, à ouvrir une porte...; c'est ce qui se fait avec «l'aménagement » qui change le «contenu »

- Soit il s'agit du renforcement des fondations nécessaires pour permettre d'ajouter un étage... C'est ce que fait « la mutation » en changeant les interactions.

\section{L'EVALUATION CIRCULAIRE}

Comme tous les systèmes, les organisations évoluent dans le temps.

Même si une hypothèse les considère comme opérantes à leur création, tout au long de leur vie, elles vont évoluer. Il est illusoire de croire que cet état ne sera pas modifié. L'observation a montré que toutes passent par des phases inopérantes, qui si elles ne sont pas corrigées, conduiront l'organisation à stagner ou régresser, voire même disparaître.

Le rôle de l'évaluation circulaire (Piecq, 2011) est de leur permettre d'évoluer par le dépistage de leurs difficultés et d'intervenir à temps avant qu'elles ne se transforment en blocages,

Son objectif est de piloter l'organisation dans la durabilité.

L'évaluation circulaire est un processus de régulation qui évalue régulièrement le fonctionnement de l'organisation en

- ne travaillant pas directement sur les blocages ;

- s'appuyant sur les PDI ; 
- diagnostiquant l'état dans lequel l'organisation se trouve ;

- relevant les symptômes ;

- élaborant des stratégies en fonction du diagnostic qui :

1) respecterons la structure de base de l'organisation en utilisant l'aménagement

2) changeront la structure de base de l'organisation en utilisant la mutation pour élabore une nouvelle structure.

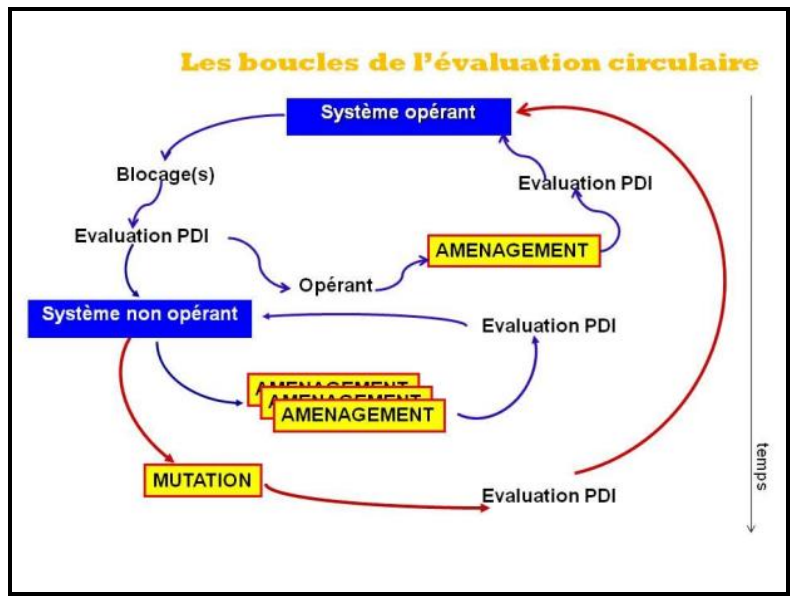

\section{CONCLUSIONS}

L'évaluation circulaire conduit à diagnostiquer régulièrement l'organisation. C'est cette régularité qui permet de résoudre efficacement les difficultés de l'organisation, en adaptant les stratégies à utiliser pour qu'elle ne rate pas sa cible, pour qu'elle atteigne sa finalité, pour que ses interactions soient modifiées et pour s'adapter aux changements de contextes.

De plus si des difficultés n'ont pas été solutionnées par les stratégies d'aménagements elles vont s'enkyster devenir des impasses et le problème. L'évaluation circulaire conduit à élaborer la stratégie adéquate : la mutation.

Toutes les organisations rencontrent des difficultés au cours de leur cheminement, elles ne sont pas un «long fleuve tranquille ». L'observation montre qu'au cours du temps, les organisations humaines et non humaines même lorsqu'elles croient atteindre leur apogée doivent modifier leur structure pour survivre ou elles disparaitront au profit d'autres. On pourrait dire que c'est ce qui est arrivé aux animaux préhistoriques qui n'ont pas pu muter lors des changements de climats.

"L'évaluation circulaire est un processus qui explore la vie des organisations qui analogiquement, peut être considéré comme un processus d'évaluation de LA VIE. Il observe le présent, interroge le passé, et grâce à une spirale infinie d'hypothèses, d'interrogations, de réflexions, d'élaborations et de transformations, se projette dans l'infini de l'avenir" (Piecq, 2011) ; elle est un processus qui explore la vie des organisations pour les inscrire dans la durabilité.

\section{BIBLIOGRAPHIE}

Ashby, W.R. (1958). Introduction à la Cybernétique, Dunod, Paris.

Fisch, R., Weakland, J., Watzlawick, P. (1975). Changements paradoxes et psychothérapie, Paris : Le Seuil.

Guattari F. (1975). "L’hétérogenèse machinique". Chimère, n¹1, repris dans Chaosmose, Galilée, 1992 Seuil.

Maturana, H., Varela, F. (1980). Autopoiesis and Cognition: The realization of the Living, Boston. 
Piecq, A., (2011). De la pensée systémique à la pratique de l'organisation - "Le giroscope". L'Harmattan, Paris.

Prigogine, I., Stengers, I. (1986). La Nouvelle alliance, Gallimard.

Prigogine, I., Glansdorff, P. (1971). Structure, stabilité et fluctuations. Masson.

von Bertalanffy, L. (1973). La Théorie générale des systèmes, Dunod, Paris.

Watzlawick, P., Beavin, J.H., Jackson, D.D. (1972). Une logique de la communication. Le Seuil. 
